\section{Sun sets on launch monopoly}

Tokyo

DRASTIC reorganization is on the way for the National Space Development Agency (NASDA), the larger of Japan's two space organizations, after Japan last week gave in to US demands for greater access to its satellite market.

The dispute centred on a new generation of communication satellites (CS-4) that NASDA planned to develop for launch in the mid-1990s with substantial financial support from Nippon Telephone and Telegraph (NTT), Japan's partly privatized domestic telecommunications organization. Two CS-4 satellites were to be launched and used by NTT.

The United States argued that CS-4 is a commercial project and should be open to foreign bidding. Japan countered with the view that it is a non-commercial research and development project.

But last week, Japanese negotiators in Washington agreed to revise the CS-4 project completely. NTT will no longer participate and is free to buy foreign-made telecommunications satellites (something the company has long wanted to do because US satellites are much cheaper than those made in Japan). Only one CS-4 satellite will be built by NASDA and its development will be merged with that of

\section{Smaller greenhouse satellites?}

\section{Washington}

NASA (the National Aeronautics and Space Administration)'s plan to launch two large satellites at the end of the decade to monitor the impact of the greenhouse effect may miss key measurements, the National Academy of Sciences said last week in a preliminary review of the US global change research programme.

Several smaller satellites could make simultaneous measurements at different altitudes and positions, allowing researchers to observe the climate as a whole, the academy points out. A single large satellite makes it necessary for all the onboard instruments to fly in the same orbit and increases the risk that a sensor failure could result in long gaps between measurements. Soil moisture and global biomass may also not be adequately measured because the only instrument that can monitor them from space - synthetic aperture radar (SAR) - is not included in the NASA Earth Observing System (EOS) plans, the academy said. Because SAR instruments are not compatible with the planned EOS satellites, the academy recommends a free-flying SAR mission.

\section{G. Christopher Anderson}

an Experimental Data Relay and Tracking Satellite (EDRTS) to make the project less commercial in nature.

The agreement affects much more than just the CS-4 project. Japan has agreed that all government-procured commercial satellites, including broadcasting and meteorological satellites, will now be open to foreign bidding and NASDA will be restricted to the production and launch of research satellites. Fifty per cent of the satellite projects planned by NASDA will now be abandoned, including a geostationary meteorological satellite (GMS-5) and a new generation of broadcasting satellites (BS-4), according to Katsuo Yonezawa of NASDA's international division.

Although loss of these satellites could cut drastically into NASDA's budget, Yonezawa says that more money will probably be directed into NASDA's participation in the US space station project and "other new research projects". Agency officials are also worried about loss of payload for the new $\mathrm{H}-2$ rocket which is currently scheduled for first launch in 1993. The satellites that will have to be abandoned by NASDA would have been among the first to be launched by the $\mathrm{H}-2$.

NASDA officials are hoping that the $\mathrm{H}$ 2 will be used to launch some US scientific payloads instead. An agreement has already been reached with the US National Aeronautics and Space Administration (NASA) to launch a US satellite for monitoring tropical rainfall (TRMM) with the $\mathrm{H}-2$ in 1996 . The $\mathrm{H}-2$ may also be used to launch some of a series of US life science satellites (LifeSat) in the mid1990s.

Last week's agreement will require revision of Japan's space policy guidelines, according to Yonezawa. He says that it is not yet clear who will be responsible for the launch of commercial satellites such as broadcasting and meteorological satellites although they may go to the European Space Agency's Ariane launch vehicle or other foreign commercial launch vehicles.

With NASDA responsible for only research satellites, the distinction between NASDA and its smaller brother, the Institute of Space and Astronautical Science (ISAS), which launches scientific payloads, will blur. But as the two space agencies are attached to different government organizations - NASDA to the Science and Technology Agency (STA) and ISAS to the Ministry of Education, Science and Culture (MESC) - it is unlikely that the two will be merged. Instead, NASDA will probably concentrate on developing manned space activities while ISAS will focus on small scientific interplanetary missions.

David Swinbanks
Rocket soars as science is attacked

\section{Tel Aviv}

As Offeq II, Israel's second satellite, was launched into space on an Israeli rocket last week, the country's civil service commissioner called for the closure of the Ministry of Science and Technology, along with four others, as a "money-saving measure".

Meir Gabai, who is responsible for Israel's 45,000 civil servants, said there was "no justification" for the large number of ministries (22) in Israel. He pointed out that Switzerland has just seven ministries. But Arye Shumer, director-general of the Ministry of Science and Technology, retorted that unless the Science Ministry "becomes one of the five ministries with the highest priority and budget, science will continue to decline". Shumer attacked the Treasury for not allocating enough to science in the latest budget, finalized in the Knesset earlier this month. But he also said that the $\$ 600$ million budget for civilian and military research and development would be "almost enough" if one body, "preferably the Science Ministry", could coordinate its distribution. Shumer also criticized "the 15 professors who sit in the Knesset" for not doing enough to advance the cause of science.

One of those professors, Yuval Ne'eman, chairman of the Israel Space Agency, spent last week denying reports that Offeq II is a spy satellite. He insists that its mission is purely scientific. The $160-\mathrm{kg}$ satellite has the same dimensions as its predecessor (launched in September 1988) but has a larger memory and the capability of twoway communication. Both were launched with a solid-fuel Shavit rocket, which Ne'eman has compared with the Chinese Long March or the Vostek SA3, but Offeq II is expected to have a shorter lifespan than the four months of Offeq $I$. The next in the series, Offeq III, will be launched in 1992.

Lisa Periman

\section{Pegasus flies}

\section{Washington}

Pegasus, the first privately developed US rocket to carry a payload into space, was successfully launched from a B-52 bomber last week. The 10 -minute flight carried into orbit a Navy communications satellite and a 422-pound National Aeronautics and Space Administration (NASA) payload that measured launch vehicle conditions. At a cost of $\$ 6.5$ million a launch, the flight marks a promising beginning for the US effort to find a cheap and reliable way of sending small satellites into space. Because it is launched by an aircraft, the $\mathbf{5 0 - f o o t}$ winged rocket can put payloads into orbit at a cost of about $\$ 6,000$ a pound, compared with about $\$ 20,000$ a pound for ground-launched rockets.

G. Christopher Anderson 